\title{
A new system for naming ribosomal proteins
}

Nenad Ban ${ }^{1}$, Roland Beckmann ${ }^{2}$, Jamie HD Cate ${ }^{3}$, Jonathan D Dinman ${ }^{4}$, François Dragon ${ }^{5}$, Steven R Ellis ${ }^{6}$, Denis LJ Lafontaine ${ }^{7}$, Lasse Lindahl ${ }^{8}$, Anders Liljas ${ }^{9}$, Jeffrey M Lipton ${ }^{10,11}$, Michael A McAlear ${ }^{12}$, Peter B Moore ${ }^{13}$, Harry F Noller ${ }^{14}$, Joaquin Ortega ${ }^{15}$, Vikram Govind Panse $^{16}$, V Ramakrishnan ${ }^{17}$, Christian MT Spahn ${ }^{18}$, Thomas A Steitz ${ }^{19}$, Marek Tchorzewski $^{20}$, David Tollervey ${ }^{21}$, Alan J Warren ${ }^{17}$, James R Williamson ${ }^{22}$, Daniel Wilson $^{23}$, Ada Yonath ${ }^{24}$, and Marat Yusupov 25

${ }^{1}$ Institute of Molecular Biology and Biophysics, ETH Zurich, Schafmattstrasse 30, 8093 Zurich, Switzerland ${ }^{2}$ Gene Center and Center for Integrated Protein Science Munich, Department of Biochemistry, Feodor-Lynen Str. 25, University of Munich, 81377 Munich, Germany ${ }^{3}$ Department of Chemistry, University of California, Berkeley, CA 94720, USA ${ }^{4}$ Department of Cell Biology and Molecular Genetics, University of Maryland, College Park, MD 20742, USA 5Département des sciences biologiques and Centre de recherche BioMed, Université du Québec à Montréal, Montréal, Québec, Canada ${ }^{6}$ Department of Biochemistry and Molecular Biology, University of Louisville, Louisville, KY 40202, USA ${ }^{7}$ RNA Molecular Biology, FRS/F.N.R.S., Université Libre de Bruxelles, Charleroi Campus, B-6041 Charleroi, Belgium ${ }^{8}$ Biological Sciences, University of Maryland Baltimore County, Baltimore, MD 21250, USA ${ }^{9}$ Biochemistry and Structural Biology, Lund University, Åkeröv 26, SE-793 Leksand, Sweden ${ }^{10}$ Feinstein Institute for Medical Research, 350 Community Dr., Manhasset, NY 11030, USA ${ }^{11}$ Hofstra School of Medicine, 500 Hofstra University, Hempstead, NY 11549, USA ${ }^{12}$ Molecular Biology and Biochemistry Department, Wesleyan University, 237 Church St., Middletown, CT 06459, USA ${ }^{13}$ Department of Chemistry, Yale University, PO Box 208107, New Haven, CT 06520, USA ${ }^{14}$ Department of MCD Biology, UCSC, Santa Cruz, CA 94720, USA ${ }^{15}$ Department of Biochemistry and Biomedical Sciences, DeGroote Institute for Infectious Diseases Research, McMaster University, 1280 Main Street West, Hamilton, Ontario L8S 4K1, Canada ${ }^{16}$ Institute for Biochemistry, ETH Zurich, HPM F12.2, Otto-Stern-Weg 3, CH-8093 Zurich, Switzerland ${ }^{17} \mathrm{MRC}$ Laboratory of Molecular Biology, Francis Crick Ave., Cambridge Biomedical Campus, Cambridge CB2 0QH, UK ${ }^{18}$ Institut für Medizinsche Physik und Biophysik, Charite-Universitätsmedizin, Ziegelstrass 5-6, 10117 Berlin, Germany ${ }^{19}$ Department of Molecular Biophysics and Biochemistry, Yale University, PO Box 208114, New Haven, CT 06520, USA ${ }^{20}$ Department of Molecular Biology, Maria Curie-Sklodowska University, Akademicka 19, 20-033 Lublin, Poland ${ }^{21}$ Welcome Trust Centre for Cell Biology, University of Edinburgh, Edinburgh EH9 3JR, UK ${ }^{22}$ Department of Molecular Biology, Scripps Research Institute, La Jolla, CA 92037, USA ${ }^{23}$ Gene Center, University of Munich, Feodor-Lynen-Str. 25, 81377 Munich, Germany ${ }^{24}$ Structural Biology Department, Weizmann Institute of Science,

(C) 2014 Elsevier Ltd. All rights reserved.

Corresponding authors: Liljas, Anders (anders.liljas@mbfys.lu.se) and Moore, Peter B (peter.moore@yale.edu) and Yusupov, Marat (marat@igbmc.u-strasbg.fr).

Appendix A. Supplementary data: Supplementary material related to this article can be found, in the online version, at http:// dx.doi.org/10.1016/j.sbi.2014.01.002. 
Rehovot 76100, Israel ${ }^{25}$ Institut de Génétique et de Biologie Moléculaire et Cellulaire, 1 rue Laurent Fries, BP10142, Illkirch F-67400, France

\begin{abstract}
A system for naming ribosomal proteins is described that the authors intend to use in the future. They urge others to adopt it. The objective is to eliminate the confusion caused by the assignment of identical names to ribosomal proteins from different species that are unrelated in structure and function. In the system proposed here, homologous ribosomal proteins are assigned the same name, regardless of species. It is designed so that new names are similar enough to old names to be easily recognized, but are written in a format that unambiguously identifies them as 'new system' names.
\end{abstract}

\title{
Introduction
}

We take it as given that homologous macromolecules that perform the same functions in different organisms should be assigned the same name. Homologous macromolecules are the products of genes that have evolved from a common ancestor. The fact that two macromolecules are homologous can often be established simply by comparing their sequences, but sometimes it becomes apparent only after their three-dimensional structures have been determined so that comparisons can be done using structure-based sequence alignments.

It has long been a challenge to devise a system for naming ribosomal proteins that respects this principle. The reason is that the characterization of ribosomal proteins began in the 1960s, at a time when there were no structures and the only way to obtain protein sequences was by sequencing them directly, an enterprise that in those days could consume hundreds of milligrams of pure protein and many man years of labor. By the time enough sequences had been obtained to begin identifying homologies, several different conventions for naming ribosomal proteins had become embedded in the literature.

Here we propose a new naming system that we hope will ultimately replace its predecessors. We know that at first many will be disinclined to use it because they find it disruptive, but we hope that the logic behind it will ultimately carry the day. We view this as a sensible next step in a process that has been moving forward for over 40 years.

\section{The proposal and its historical background}

\section{The origins of the naming problem}

The naming of ribosomal proteins first emerged as a problem in the mid-1960s, when several groups began purifying and characterizing the ribosomal proteins from Escherichia coli. Each laboratory devised its own naming system, which made it hard even for members of that in-group to make sense of the data being published, let alone for anyone else. That chaos ended in 1971 when a standard experimental method for identifying these proteins was agreed upon, as well as a naming system [1]. Henceforth, ribosomal proteins from the 
small subunit would bear names having the form SX, where $\mathrm{X}$ is an integer, and ribosomal proteins from the large subunit would be designated LY, where $\mathrm{Y}$ is an integer.

The ribosomal proteins from E. coli were the first to be fully sequenced, and later on, as sequences for the ribosomal proteins from other eubacterial species accumulated, it became obvious that they all have homologs in E. coli. Thus the naming system devised for E. coli could be used for those molecules too. This practice was validated decades later when atomic resolution crystal structures appeared for the ribosomes and ribosomal subunits from E. coli [2], Thermus thermophilus [3,4], and Deinococcus radiodurans [5], all of which are eubacteria. As expected, ribosomal proteins that had been identified by sequence as homologs turned out to have similar three-dimensional structures, and to bind to their respective ribosomes in equivalent locations and in the same way. They are functionally equivalent.

By the mid-1980s, it was obvious that the protein-naming problem that had plagued the part of the ribosome community interested in E. coli in the 1960s was fast becoming an even larger issue for those concerned with archaeal and eukaryotic ribosomal proteins. Not surprisingly, names were being assigned to these proteins before their sequences were determined, and unfortunately, those names usually had the form SX or LY, with a prefix sometimes added to identify the species of origin. In many instances, at the time names were assigned, the proteins in question were nothing more than spots on a two-dimensional gel as far as biochemists were concerned. Furthermore several different naming systems were developed for yeast ribosomal proteins, a sad echo of the situation that had prevailed in the E. coli community a decade or two earlier. The probability that two proteins having the same 'S' or ' $L$ ' name that had been obtained from different species within either kingdom would be homologous was modest. The probability that either would be homologous to the eubacterial ribosomal protein of the same name, if there was one, was next to none. Nevertheless, almost as soon as sequences became available it became clear that the ribosomal proteins obtained from different archaea are homologous, as are the ribosomal proteins isolated from different eukaryotes.

\section{Progress towards a rational naming system}

By the late 1980s, the number of sequences for ribosomal proteins available had become large enough so that homologies could be confidently identified across kingdom boundaries. In 1989, Wittmann-Liebold and her collaborators published the results of an extensive set of cross-kingdom sequence comparisons [6]. Their work demonstrated that a substantial fraction of the ribosomal proteins from the archaean Haloarcula marismortui are homologous to ribosomal proteins from the eubacterium E. coli, and that the rest appeared to be homologous to eukaryotic ribosomal proteins. They proposed that in the future, the ribosomal proteins from $H$. marismortui that have eubacterial homologs be designated using the names of their eubacterial homologs. Six years later, the results of an even more comprehensive set of sequence comparisons appeared that confirmed the Berlin group's conclusions about $H$. marismortui, identified the rat homologs of all the ribosomal proteins from that organism that lack eubacterial homologs, identified the rat ribosomal proteins that have eubacterial homologs, and related the ribosomal proteins of yeast to those of the rat [7]. 
Not long thereafter a new naming system for yeast ribosomal proteins (Saccharomyces cerevisiae) was proposed that assigned yeast proteins the same names as their rat homologs to the maximum extent possible. This effectively ended many of the naming problems that had grown up in the eukaryotic literature; it was a major step forward [8].

In 2000, the Yale group that solved the crystal structure of the large ribosomal subunit from H. marismortui had to take a stand on the way archaeal ribosomal proteins are named [9]. They elected to use E. coli names for the proteins in their structure Wittmann-Liebold's group had determined are homologous to E. coli proteins, and to use rat names for the rest, as both Wittmann-Liebold and Wool had suggested earlier. The objective was to make it easy for readers to access the literature relevant to those proteins, most of which describes work done with proteins that were not obtained from H. marismortui.

The same problem rose again in 2011, when the first atomic resolution crystal structures appeared for eukaryotic ribosomes [10-12]. Here there was a parting of the ways. The group in Zurich elected to use the names that had been assigned the proteins in their particles by those who had annotated the genome sequence of the organism from which their particles came (Tetrahymena thermophila); they are yeast-like. The group in Strasbourg took an approach similar to that followed earlier by the Yale group. They used E. coli names to designate the proteins in the structure they had obtained for the $S$. cerevisiae ribosome that have eubacterial homologs, and used Mager et al. [8] names for the rest. Their rationale was clear. Their structure had eliminated any uncertainties there might still have been about homologies between the ribosomal proteins from yeast and E. coli.

\section{Development of a proposal for a new naming system}

In a review published in 2012, the Strasbourg group proposed that all ribosomal proteins be named using the approach they had taken to naming proteins in the yeast ribosome [13]. Their proposal is the basis for the one being advanced here. The introduction for the section of Current Opinion in Structural Biology in which the Strasbourg review appeared, which was written by AL and PBM, invited readers to post comments about the Strasbourg proposal on a blog maintained by the publisher. A modest number of comments were received, and they were all supportive.

The Strasbourg proposal was discussed at the ribosome meeting held in Napa, CA, in the summer of 2013. It was there that the idea emerged of adding a letter prefix before protein names (see below). However, those discussions revealed that any proposal for renaming ribosomal proteins was likely to be resisted by at least some members of the eukaryotic ribosome community, which was poorly represented at the meeting. Later that summer an effort was made to reach out to that community by email. (We thank Jonathan Warner for doing the work required.) The ensuing email exchanges showed that while there was some enthusiasm for this proposal in the eukaryotic community, a consensus did not exist. Several impediments to change were identified, among them a reluctance on the part of those who run the yeast sequence data bases to rename anything, and the fact that 'old system' names have become incorporated into the clinical literature that deals with the diseases caused by mutations in ribosomal proteins. 
Nevertheless, those engaged in the determination of ribosome structures at high resolution are convinced that the time has come to assign names to ribosomal proteins that make evolutionary sense, and for that reason decided to move forward anyway. We gratefully acknowledge the support this initiative has received from other quarters.

\section{The proposal}

The system for naming ribosomal proteins we advocate is described in Tables 1 and 2, which display the equivalences between this system and several of the other naming systems now in use. It is a modest modification of the proposal first advanced by the Strasbourg group.

Since the ribosomal proteins from E. coli were the first to be isolated and fully sequenced, and are described in an extensive literature, standard priority practices in the sciences dictate that their archaeal and eukaryotic homologs be assigned E. coli names. Proteins found in ribosomes from all three domains are given the prefix ' $u$ ' (for universal), which is followed by their E. coli names. Bacterial proteins without eukaryotic (or archaeal) homologues are designated using the prefix ' $b$ ' (for bacterial). Similarly, archaeal ribosomal proteins lacking homologs in both eubacterial ribosomes and eukaryotic ribosomes are to be identified by the prefix ' $a$ ' (for archaeal), but so far none has been found. Those eukaryotic ribosomal proteins that have no eubacterial homolog, of which there are many, are given the name assigned them by Wool and his colleagues if they were first sequenced in rat (see [7]), or if they were first sequenced by the yeast community, they are given yeast names using the system first described in 1997 [8]. (Fortunately, these two naming systems are consistent with each other.) By adding the letter 'e' (for eukaryotic) before the eukaryotic-only names, the problems that would otherwise arise because of accidental overlaps in protein numbering schemes are averted, and the reader is put on notice that the proteins in question have no eubacterial homolog.

Text files with PyMOL scripts that display the Protein Data Bank (PDB) coordinates of representative eukaryotic, bacterial, archaeal and mitochondrial ribosomes, with ribosomal proteins labelled according to the old and new nomenclature, are available as supporting online material.

\section{Discussion}

Some further comments are in order. The protein in eukaryotic ribosomes that is equivalent to protein L10 in bacteria is somewhat larger, and is referred to in the literature as P0. We propose that the name uL10 be assigned to this molecule. Furthermore, only bacteria have proteins that correspond to the protein called L7/L12 in E. coli. In addition the acetylated variant of L12, L7, is not found in all bacterial species. Therefore we suggest that this protein be called bL12 unless its acetylated form is being discussed in which case it could be called bL7. In eukaryotes the proteins that have the same function as bL12, but which are not homologous to it, are called P1 and P2. In yeast, multiple forms of this protein are found: P1A, P1B, P2A, and P2B. Sometimes there is also a variant called P3, which is found exclusively in plants. We suggest that these names be retained. Furthermore, we suggest that capital letters following protein names be used to distinguish different isoforms of the same 
protein, when appropriate. The functional equivalent of bL12 in archaea has been called L12, but this is inappropriate since in sequence, that protein is closely related to P1, but not at all to bL12. Since there is only one variant we suggest that it can be called P1.

We note that the use of lower case prefixes before LY and SX names is a departure from prior practice that should make it easy for readers to distinguish names consistent with the proposal being advanced here from all of their older predecessors. In addition, this convention should make it easy to deal with the ribosomes from the mitochondria of higher eukaryotes, which have a larger number of ribosomal proteins than cytoplasmic ribosomes [14]. Well-resolved structures of these ribosomes will be needed before one can safely propose names for these proteins that are consistent with the system described here. Clearly the proteins from mitochondria ribosomes that are not homologous to cytoplasmic ribosomal proteins could be designated using the prefix ' $\mathrm{m}$ ' to distinguish them from the cytoplasmic ribosomal proteins that happen to have the same SX or LY name. To avoid ambiguities, the suffix ' $m$ ' should be added to the names of mitochondrial ribosomal proteins that have homologs in the cytosol. In this case, the suffix ' $m$ ' indicates cellular location, not taxonomic distribution. Thus, for example, uL2 would designate a particular ribosomal protein in the cytoplasm of a eukaryotic cell and the homolog of that protein found in the mitochondria of the same cell would be uL2m. An analogous naming convention could be used for chloroplast ribosomal proteins (uL2c).

We have no illusions that this proposal will forever solve all ribosomal protein naming problems. However, we do believe that adoption of the system proposed here will in the long run help clarify the already dauntingly large literature that deals with these fascinating molecules.

\section{Supplementary Material}

Refer to Web version on PubMed Central for supplementary material.

\section{References}

1. Wittmann HG, Stoeffler G, Hindennach I, Kurland CG, Randall-Hazelbauer L, Birge EA, Nomura M, Kaltschmidt E, Mizushima S, Traut RR, et al. Correlation of 30S ribosomal proteins of Escherichia coli isolated in different laboratories. Mol Gen Genetics. 1971; 111:327-333.

2. Schuwirth BS, Borovinskaya MA, Hau CW, Zhang W, Vila-Sanjuro A, Holton JH, Doudna Cate JH. Structure of the bacterial ribosome at 3.5 A resolution. Science. 2005; 310:827-834. [PubMed: 16272117]

3. Wimberly BT, Brodersen DE, Clemons WM, Morgan-Warren RJ, Carter AP, Vonrhein C, Hartsch T, Ramakrishnan V. Structure of the 30S ribosomal subunit. Nature. 2000; 407:327-339. [PubMed: 11014182]

4. Yusupov MM, Yusupova GZ, Baucom A, Lieberman K, Earnest TN, Cate JHD, Noller HF. Crystal structure of the ribosome at 5.5 A resolution. Science. 2001; 292:883-896. [PubMed: 11283358]

5. Harms J, Schluenzen F, Zarivach R, Bashan A, Gat S, Agmon I, Bartels H, Franceschi F, Yonath A. High resolution structure of the large ribosomal subunit from a mesophilic eubacterium. Cell. 2001; 107:679-688. [PubMed: 11733066]

6. Wittmann-Liebold, B.; Kopke, AKE.; Arndt, E.; Kromer, W.; Hatakeyama, T.; Wittmann, HG. Sequence comparison and evolution of ribosomal proteins and their genes. In: Hill, WE.; Dahlberg, 
A.; Garrett, RA.; Moore, PB.; Schlessigner, D.; Warner, JR., editors. The Ribosome Structure, Function, \& Genetics. American Society for Microbiology; 1990. p. 598-616.

7. Wool IG, Chan YL, Gluck A. Structure and evolution of mammalian ribosomal proteins. Biochem Cell Biol. 1995; 73:933-947. [PubMed: 8722009]

8. Mager WH, Planta RJ. A new nomenclature for the cytoplasmic ribosomal proteins of Saccharomyces cerevisiae. Nucl Acid Res. 1997; 25:4872-4875.

9. Ban N, Nissen P, Hansen J, Moore PB, Steitz TA. The complete atomic structure of the large ribosomal subunit at 2.4 A resolution. Science. 2000; 289:905-920. [PubMed: 10937989]

10. Ben-Shem A, de Loubresse NG, Melnikov S, Jenner L, Yusopova G, Yusopov M. The structure of the eukaryotic ribosome at 3.0 A resolution. Science. 2011; 334:1524-1529. [PubMed: 22096102]

11. Klinge S, Voigts-Hoffmann F, Leibundgut M, Arpagaus S, Ban N. Crystal structure of the eukaryotic 60S ribosomal subunit in complex with initiation factor 6. Science. 2011; 334:941-948. [PubMed: 22052974]

12. Rabl J, Leibundgut M, Ataide SF, Haag A, Ban N. Crystal structure of the eukaryotic 40S ribosome in complex with intitation factor 1. Science. 2011; 331:730-736. [PubMed: 21205638]

13. Jenner L, Melnikov S, Garreau de Loubresse N, Ben-Shem A, Isakova M, Urzhumtsev A, Meskauskas A, Dinman J, Yusopova G, Yusupov M. Crystal structure of the 80S yeast ribosome. Curr Opin Struct Biol. 2012; 22:759-767. [PubMed: 22884264]

14. Desmond E, Brochier-Armanet C, Forterre P, Gribaldo S. On the last common ancestor and early evolution of eukaryotes: reconstructing the history of mitochondrial ribosomes. Res Microbiol. 2011; 162:53-70. [PubMed: 21034815] 
Table 1

New nomenclature for proteins from the small ribosomal subunit

\begin{tabular}{|c|c|c|c|c|}
\hline New name ${ }^{\#}$ & Taxonomic range* & Bacteria name & Yeast name & Human name \\
\hline $\mathrm{bS} 1$ & B & S1 & - & - \\
\hline eS1 & A E & - & S1 & S3A \\
\hline uS2 & B A E & S2 & So & SA \\
\hline uS3 & B A E & S3 & S3 & S3 \\
\hline uS4 & B A E & S4 & S9 & S9 \\
\hline eS4 & A E & - & S4 & S4 \\
\hline uS5 & B A E & S5 & $\mathrm{S} 2$ & $\mathrm{~S} 2$ \\
\hline bS6 & $\mathrm{B}$ & S6 & - & - \\
\hline eS6 & A E & - & S6 & S6 \\
\hline uS7 & B A E & S7 & S5 & S5 \\
\hline eS7 & $\mathrm{E}$ & - & S7 & $\mathrm{S} 7$ \\
\hline uS8 & B A E & S8 & S22 & $\mathrm{S} 15 \mathrm{~A}$ \\
\hline eS8 & A E & - & S8 & $\mathrm{S} 8$ \\
\hline uS9 & B A E & S9 & S16 & S16 \\
\hline uS10 & B A E & $\mathrm{S} 10$ & S20 & S20 \\
\hline eS10 & $\mathrm{E}$ & - & $\mathrm{S} 10$ & $\mathrm{~S} 10$ \\
\hline uS11 & B A E & S11 & S14 & S14 \\
\hline uS12 & B A E & $\mathrm{S} 12$ & S23 & S23 \\
\hline eS12 & $\mathrm{E}$ & - & S12 & $\mathrm{S} 12$ \\
\hline uS13 & B A E & $\mathrm{S} 13$ & $\mathrm{~S} 18$ & $\mathrm{~S} 18$ \\
\hline uS14 & B A E & S14 & S29 & S29 \\
\hline uS15 & B A E & S15 & S13 & $\mathrm{S} 13$ \\
\hline bS16 & B & S16 & - & - \\
\hline uS17 & B A E & S17 & S11 & S11 \\
\hline eS17 & $\mathrm{A} \mathrm{E}$ & - & S17 & S17 \\
\hline bS18 & B & $\mathrm{S} 18$ & - & - \\
\hline uS19 & B A E & S19 & S15 & S15 \\
\hline eS19 & A E & - & S19 & S19 \\
\hline bS20 & B & S20 & - & - \\
\hline bS21 & B & S21 & - & - \\
\hline bTHX & B & THX & - & - \\
\hline eS21 & $\mathrm{E}$ & - & S21 & S21 \\
\hline eS24 & $\mathrm{AE}$ & - & S24 & S24 \\
\hline eS25 & A E & - & S25 & S25 \\
\hline eS26 & $\mathrm{E}$ & - & S26 & S26 \\
\hline eS27 & A E & - & S27 & S27 \\
\hline eS28 & A E & - & S28 & S28 \\
\hline eS30 & A E & - & S30 & $\mathrm{S} 30$ \\
\hline eS31 & A E & - & $\mathrm{S} 31$ & S27A \\
\hline
\end{tabular}




\begin{tabular}{lllll}
\hline New name $^{\#}$ & Taxonomic range $^{*}$ & Bacteria name & Yeast name & Human name \\
\hline RACK1 & E & - & Asc1 & RACK1 \\
\hline
\end{tabular}

\# b: bacterial, e: eukaryotic, u: universal.

B: bacteria, A: archaea, E: eukaryotes. 
Table 2

Nomenclature for proteins from the large ribosomal subunit

\begin{tabular}{|c|c|c|c|c|}
\hline New name ${ }^{\#}$ & Taxonomic range ${ }^{*}$ & Bacteria name & Yeast name & Human name \\
\hline $\mathrm{uL1}$ & B A E & L1 & L1 & L10A \\
\hline uL2 & B A E & $\mathrm{L} 2$ & $\mathrm{~L} 2$ & L8 \\
\hline uL3 & B A E & L3 & L3 & L3 \\
\hline uL4 & B A E & L4 & L4 & L4 \\
\hline uL5 & B A E & L5 & L11 & L11 \\
\hline uL6 & B A E & L6 & L9 & L9 \\
\hline eL6 & $\mathrm{E}$ & - & L6 & L6 \\
\hline eL8 & A E & - & L8 & L7A \\
\hline bL9 & B & L9 & - & - \\
\hline uL10 & B A E & L10 & $\mathrm{P} 0$ & $\mathrm{P} 0$ \\
\hline uL11 & B A E & L11 & L12 & $\mathrm{L} 12$ \\
\hline bL12 & B & L7/L12 & - & - \\
\hline uL13 & B A E & L13 & L16 & L13A \\
\hline eL13 & A E & - & $\mathrm{L} 13$ & $\mathrm{~L} 13$ \\
\hline uL14 & B A E & L14 & L23 & $\mathrm{L} 23$ \\
\hline eL14 & A E & - & L14 & L14 \\
\hline uL15 & B A E & L15 & $\mathrm{L} 28$ & L27A \\
\hline eL15 & A E & - & L15 & L15 \\
\hline uL16 & B A E & L16 & L10 & L10 \\
\hline bL17 & B & L17 & - & - \\
\hline uL18 & B A E & L18 & L5 & L5 \\
\hline eL18 & $\mathrm{AE}$ & - & L18 & L18 \\
\hline bL19 & B & L19 & - & - \\
\hline eL19 & A E & - & L19 & L19 \\
\hline bL20 & B & L20 & - & - \\
\hline eL20 & $\mathrm{E}$ & - & L20 & L18A \\
\hline bL21 & B & L21 & - & - \\
\hline eL21 & A E & - & L21 & L21 \\
\hline uL22 & B A E & L22 & L17 & L17 \\
\hline eL22 & $\mathrm{E}$ & - & L22 & L22 \\
\hline uL23 & B A E & L23 & L25 & L23A \\
\hline uL24 & B A E & L24 & L26 & L26 \\
\hline eL24 & A E & - & L24 & L24 \\
\hline bL25 & B & L25 & - & - \\
\hline bL27 & B & L27 & - & - \\
\hline eL27 & $\mathrm{E}$ & - & L27 & L27 \\
\hline bL28 & B & L28 & - & - \\
\hline eL28 & $\mathrm{E}$ & - & - & L28 \\
\hline uL29 & B A E & L29 & L35 & L35 \\
\hline
\end{tabular}




\begin{tabular}{lllll}
\hline New name $^{\#}$ & Taxonomic range & Bacteria name & Yeast name & Human name \\
\hline eL29 & E & - & L29 & L29 \\
uL30 & B A E & L30 & L7 & L7 \\
eL30 & A E & - & L30 & L30 \\
bL31 & B & L31 & - & - \\
eL31 & A E & - & L31 & L31 \\
bL32 & B & L32 & - & - \\
eL32 & A E & - & L32 & L32 \\
bL33 & B & L33 & - & - \\
eL33 & A E & - & L33 & L35A \\
bL34 & B & L34 & - & - \\
eL34 & A E & - & L34 & L34 \\
bL35 & B & L35 & - & - \\
bL36 & B & L36 & - & - \\
eL36 & E & - & L36 & L36 \\
eL37 & A E & - & L37 & L37 \\
eL38 & A E & - & L38 & L38 \\
eL39 & A E & - & L39 & L39 \\
eL40 & A E & - & L40 & L40 \\
eL41 & A E & - & L41 & L41 \\
eL42 & A E & A E & - & L36A \\
eL43 & A E & - & L37A \\
P1/P2 & & - & P1/P2 (a $\beta)$ \\
\hline & & - &
\end{tabular}

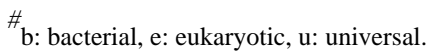

* B: bacteria, A: archaea, E: eukaryotes. 